\title{
$\mathrm{Mg}$ 掺杂 $\mathrm{GaN}$ 纳米线的结构及其特性
}

\author{
薛成山* 张冬冬庄惠照黄英龙王邹平王英 \\ (山东师范大学物理与电子科学学院半导体研究所, 济南 250014)
}

\begin{abstract}
摘要：利用类似 Delta 掺杂技术在硅衬底上沉积 $\mathrm{Mg}: \mathrm{Ga}_{2} \mathrm{O}_{3}$ 薄膜, 然后在 $850{ }^{\circ} \mathrm{C}$ 下对薄膜进行氨化, 反应后制备 出大量 $\mathrm{Mg}$ 掺杂 $\mathrm{GaN}$ 纳米线. 采用扫描电子显微镜 $(\mathrm{SEM}) 、 X$ 射线衍射 $(X R D)$ 、傅里叶变换红外(FTIR)光谱和高 分辨透射电子显微镜(HRTEM)对样品进行分析. 结果表明, $\mathrm{Mg}$ 掺杂 $\mathrm{GaN}$ 纳米线具有六方纤锌矿单晶结构, 纳米 线的直径在 30-50 $\mathrm{nm}$ 范围内, 长度为几十微米.
\end{abstract}

关键词：氮化镓; 纳米线; 单晶; $\mathrm{Mg}$ 掺杂

中图分类号： O647; TN304.23

\section{Structure and Characterization of Mg-Doped GaN Nanowires}

\author{
XUE Cheng-Shan* ZHANG Dong-Dong ZHUANG Hui-Zhao \\ HUANG Ying-Long WANG Zou-Ping WANG Ying \\ (Institute of Semiconductors, College of Physics and Electronics, Shandong Normal University, Jinan 250014, P. R. China)
}

\begin{abstract}
Large-scale Mg-doped $\mathrm{GaN}$ nanowires were synthesized by ammoniating $\mathrm{Mg}: \mathrm{Ga}_{2} \mathrm{O}_{3}$ thin films at $850{ }^{\circ} \mathrm{C}$ which were deposited on the $\mathrm{Si}$ substrate using the resembling Delta doping method. These GaN nanowires were characterized by scanning electron microscopy (SEM), X-ray diffraction (XRD), Fourier transformed infrared (FTIR) spectroscopy and high resolution transmission electron microscopy (HRTEM). The results indicated that nanowires were hexagonal wurzite $\mathrm{GaN}$ single crystals. The diameter of nanowires was in the range of $35-50 \mathrm{~nm}$ with lengths of up to several tens of micrometers.
\end{abstract}

Key Words: GaN; Nanowires; Single crystal; Mg-doped

氮化镓 $(\mathrm{GaN})$ 是一种重要的 III-V 族直接带隙 宽禁带化合物半导体材料, 室温下禁带宽度为 3.39 $\mathrm{eV}$, 在常温常压下的稳定相是六方纤锌矿结构. 因 其具有高的发光效率、高的电子漂移饱和速度、高的 热导率、耐高温和抗辐射等特点, 被广泛地用来制作 各种高亮度蓝、绿光发光二极管、蓝色激光器和紫外 探测器, 同时又适于制作抗辐射、高频、高温和高密 度集成的电子器件 ${ }^{[1-4]}$. 随着这类器件的发展, 为了 更好地实现其光电子特性, 适当的掺杂是非常有必 要的. 迄今为止, 人们已经采用了很多种掺杂方法和 掺杂剂来制备掺杂 $\mathrm{GaN}$ 的一维纳米结构, 如 $\mathrm{Hg}$ 掺
杂 $\mathrm{GaN}$ 纳米线的紫外发光特性 ${ }^{[5]} 、 \mathrm{GaN}$ 纳米线的浅 掺杂能级 ${ }^{[6]} \mathrm{Mn}$ 掺杂 $\mathrm{GaN}$ 纳米线的磁特性研究 ${ }^{[7]} 、 \mathrm{Al}$ 掺 杂 $\mathrm{GaN}$ 纳米线的合成及其PL (photoluminescene)特 性 ${ }^{[8]}$ 等. 我们选择金属 $\mathrm{Mg}$ 作为 $\mathrm{GaN}$ 纳米线的掺杂 剂是 因为 $\mathrm{Mg}$ 的离子半径 $(0.065 \mathrm{~nm})$ 略大于 $\mathrm{Ga}$ 的 离子半径 $(0.062 \mathrm{~nm}), \mathrm{Mg}$ 被认为是 $p$ 型掺杂 $\mathrm{GaN}$ 的 最佳掺杂剂. 本文采用类似 Delta 掺杂技术在硅衬 底上沉积 $\mathrm{Mg}: \mathrm{Ga}_{2} \mathrm{O}_{3}$ 薄膜, 然后在 $850{ }^{\circ} \mathrm{C}$ 下对薄膜进 行氨化, 反应后制备出大量 $\mathrm{Mg}$ 掺杂 $\mathrm{GaN}$ 纳米线.

\section{1 实 验}

Received: July 17, 2008; Revised: September 16, 2008; Published on Web: November 13, 2008.

*Corresponding author. Email: xuechengshan@sdnu.edu.cn; Tel: +86531-86182624; Fax: +86531-86180017.

国家自然科学基金(90201025,90301002)资助项目

C. Editorial office of Acta Physico-Chimica Sinica 
首先, 采用JCK-500A型射频磁控溅射仪制备 $\mathrm{Mg}: \mathrm{Ga}_{2} \mathrm{O}_{3}$ 薄膜. 浌射用靶是纯度为 $99.99 \%$ 的 $\mathrm{Mg}$ 热压 靶和纯度为 $99.999 \%$ 的 $\mathrm{Ga}_{2} \mathrm{O}_{3}$ 烧结靶, 其分别采用直 流溅射(DC)和射频磁控(RF)溅射. 靶基距为 $80 \mathrm{~mm}$, 系统的背景真空度为 $9 \times 10^{-4} \mathrm{~Pa}$, 工作时通高纯 $\mathrm{Ar}$ $(99.999 \%)$, 分压为 $1.5 \mathrm{~Pa}$. 在此过程中, 采用了类似 Delta掺杂方法. 图 1 为 $\mathrm{Mg}: \mathrm{Ga}_{2} \mathrm{O}_{3}$ 薄膜的生长模型示 意图. 整个过程包含 25 个循环, $\mathrm{Mg}: \mathrm{Ga}_{2} \mathrm{O}_{3}$ 薄膜总的 溅射厚度为 $600 \mathrm{~nm}$ 左右, 溅射总时间为 $100 \mathrm{~min}$. 单 个循环过程如下: 先生长 $20 \mathrm{~nm}$ 的非故意掺杂 $\mathrm{Ga}_{2} \mathrm{O}_{3}$ (简写为un- $\mathrm{Ga}_{2} \mathrm{O}_{3}$ ), 保持工作气压不变, 同时停止浌 射 $\mathrm{Ga}_{2} \mathrm{O}_{3}$, 如此保持一段时间 $(5 \mathrm{~s})$ 再开始溅射 $\mathrm{Mg}, 2 \mathrm{~s}$. 最后, 将浌射好的 $\mathrm{Mg}: \mathrm{Ga}_{2} \mathrm{O}_{3}$ 薄膜样品放人管式炉的 恒温区进行氨化. 氨化温度和氨化时间分别为 850 ${ }^{\circ} \mathrm{C}$ 和 $25 \mathrm{~min}$. 氨化反应后, 样品呈白色线状物.

采用X射线衍射仪 (XRD, Rigaku D/max $2 \mathrm{rB}, \mathrm{Cu}$ $K_{\alpha}$ )、高分辨透射电子显微镜(HRTEM, Philip TECNA 120)和傅里叶变换红外光谱仪(FTIR,Tensor27)分别 对 $\mathrm{Mg}$ 掺杂 $\mathrm{GaN}$ 样品的结构和组分进行了分析, 并对 样品的表面形貌用扫描电镜(SEM, HitachS570)进行 观察.

\section{2 结果与讨论}

图 2 为 $\mathrm{Mg}$ 掺杂 $\mathrm{GaN}$ 样品表面形貌的 SEM 照 片. 图 2(a)是样品的低倍 SEM 图片, 整个样品表面 全部被高密度的纳米线所覆盖, 纳米线相互无序地 交叉在一起, 平铺或斜铺在衬底表面. 大多数纳米线 沿着平行于衬底的方向生长, 其表面粗䊁不光滑. 通 过对高倍放大图像的观察(如图 2(b)), 发现大部分 纳米线长度超过 $10 \mu \mathrm{m}$, 直径在 30-50 nm 范围内, 具有较高的纵横比. 表明在该条件下得到了较高质 量的 $\mathrm{Mg}$ 掺杂 $\mathrm{GaN}$ 纳米线.

$\mathrm{Mg}$ 掺杂 $\mathrm{GaN}$ 样品的 XRD图谱见图 3. 在 $2 \theta$ 为 $32.1^{\circ} 、 34.2^{\circ}$ 和 $36.4^{\circ}$ 处的衍射峰, 分别对应六方纤锌 矿 $\mathrm{GaN}$ 单晶的(100)、(002)和(101)面 ${ }^{[9]}$. 在图中没有

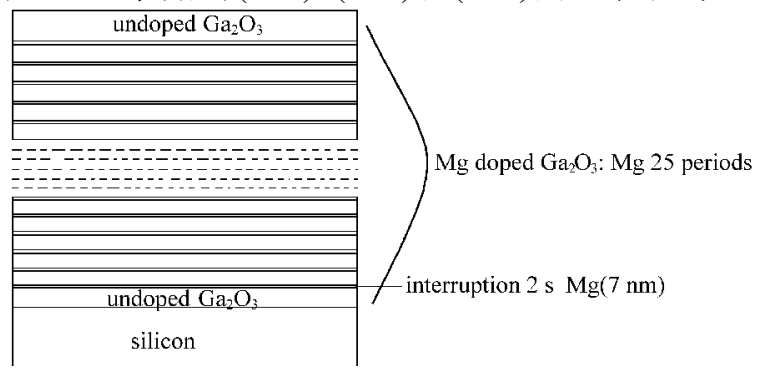

图 $1 \mathrm{Mg}: \mathrm{Ga}_{2} \mathrm{O}_{3}$ 薄膜的生长模型

Fig.1 The growth sketch of $\mathrm{Mg}: \mathrm{Ga}_{2} \mathrm{O}_{3}$ thin film
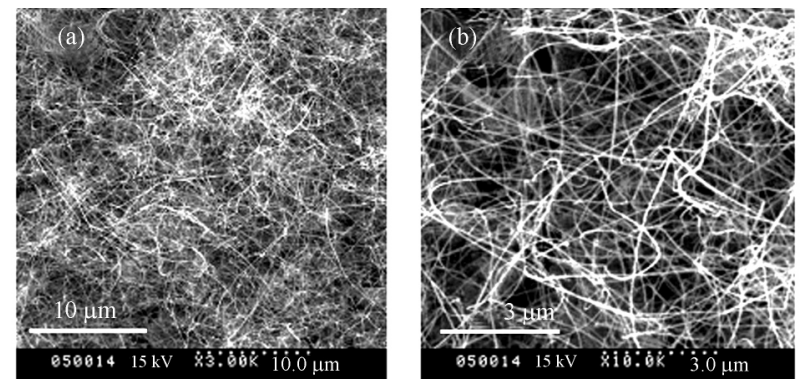

图 $2 \mathrm{Mg}$ 掺杂 $\mathrm{GaN}$ 纳米线的扫描电镜图

Fig.2 SEM images of Mg-doped GaN nanowires

发现 $\mathrm{Ga}_{2} \mathrm{O}_{3} 、 \mathrm{Mg}$ 或 $\mathrm{MgO}$ 的衍射峰, 从而表明没有 $\mathrm{Mg}$ 或者 $\mathrm{MgO}$ 覆盖在 $\mathrm{GaN}$ 纳米线的表面. 同时, $\mathrm{Mg}$ 掺杂 $\mathrm{GaN}$ 纳米线的衍射峰相比未掺杂 $\mathrm{GaN}$ 纳米线 发生了很小的蓝移. 未掺杂和 $\mathrm{Mg}$ 掺杂 $\mathrm{GaN}$ 纳米线 的(100)晶面间距分别是 0.2757 和 $0.2789 \mathrm{~nm}$. 这个结 果与 $\mathrm{Han}$ 等人 ${ }^{[10]}$ 研究 $\mathrm{Mn}$ 掺杂 $\mathrm{GaN}$ 的结果相似.

我们进一步研究了 $\mathrm{Mg}$ 掺杂 $\mathrm{GaN}$ 纳米线的内部 结构信息如图4. 图4(a)为单根GaN纳米线的TEM图, 直径为 $35 \mathrm{~nm}$, 但表面粗糙. 可能因为纳米线在富氮 条件下生长, 以及 $\mathrm{Mg}$ 的掺杂影响了生长过程中 $\mathrm{Ga}$ 的质量输运, 从而导致了粗糙表面 $\mathrm{GaN}$ 纳米线的生 成 ${ }^{[11]}$. 图中插图为该纳米线的选区电子衍射(SAED) 图像, 与六方 $\mathrm{GaN}[1 \overline{2} 1 \overline{3}]$ 的衍射一致, 进一步说明该 纳米线为六方纤锌矿结构的 $\mathrm{GaN}$ 单晶相, 与前述结 果一致. 其HRTEM晶格图像如图4(b)所示, 该纳米 线的位错和缺陷很少, 晶格边缘清晰而规则, 说明纳 米线为高度结晶的单晶体. 这可能是因为 $\mathrm{Mg}$ 的掺 杂, 使 $\mathrm{GaN}$ 的晶格常数发生了细微的变化. 该纳米线 的生长方向沿着轴向, 为 [100]晶向. 图4(c)为该纳米 线的EDX分析图. 从图中可知, 该纳米线的成分组 成约 $47 \%$ (原子分数,下同)的 $\mathrm{Ga}$, 约 $48.5 \%$ 的 $\mathrm{N}$, 以及 约 $3 \%$ 的 $\mathrm{Mg}$.

图 5 为 $\mathrm{Mg}$ 掺杂 $\mathrm{GaN}$ 纳米线的 FTIR 图谱. 图

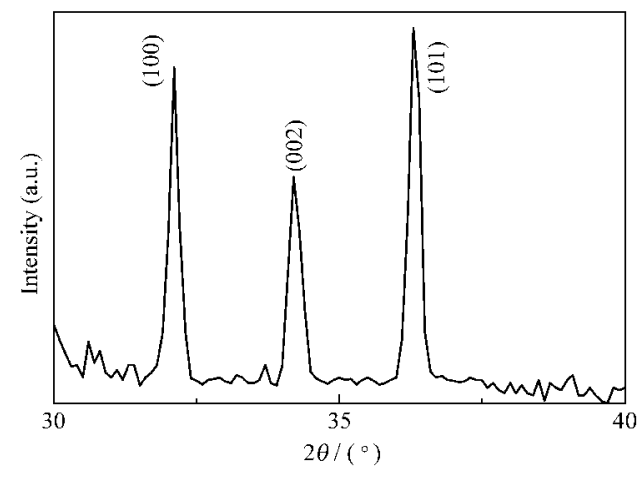

图 $3 \mathrm{Mg}$ 掺杂 $\mathrm{GaN}$ 样品的 $\mathbf{X}$ 射线衍射图谱 Fig.3 XRD pattern of the Mg-doped GaN sample 

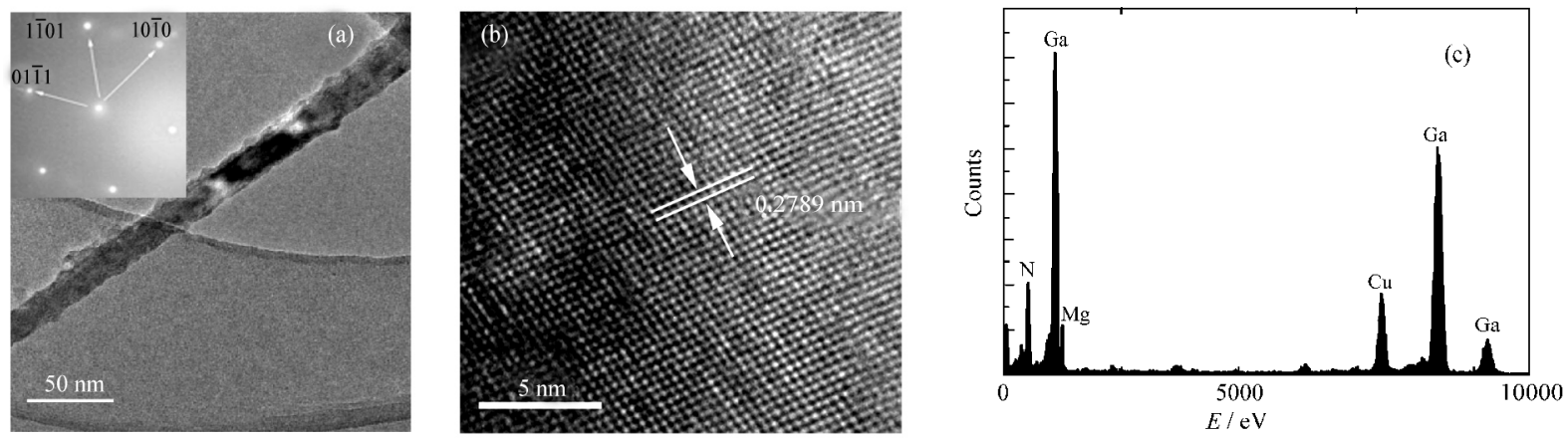

图 $4 \mathrm{Mg}$ 掺杂 GaN 纳米线的 TEM (a)、HRTEM (b)图像和 EDX 位置 (c)分析

Fig.4 TEM (a), HRTEM (b) images and EDX pattern (c) of Mg-doped GaN nanowires Inset in (a) is select area electronic diffraction (SAED) image

中存在 3 个明显的吸收带, 分别位于 $565 \mathrm{~cm}^{-1}$ (带 1$)$, $609 \mathrm{~cm}^{-1}$ (带 2)和 $1103 \mathrm{~cm}^{-1}$ (带 3). 根据文献[9,12], 带 1 对应于六方 $\mathrm{GaN}$ 晶体中 $\mathrm{Ga}-\mathrm{N}$ 键的伸缩振动 $\left[\mathrm{E}_{1}\right.$ (TO)模式], 吸收带 2 和 3 与衬底有关. 带 2 是硅晶格中 替位碳的振动吸收带 ${ }^{[13,14]}$, 带 3 对应 $\mathrm{Si}-\mathrm{O}-\mathrm{Si}$ 键的 反对称伸缩振动吸收 ${ }^{[15]}$, 是由于硅衬底表面在样品生 长或测试过程中被氧化所致.

基于上述的观察和分析, 我们可以简述其生长 过程: 在氨化过程中, 当温度超过 $800{ }^{\circ} \mathrm{C}$ 时, $\mathrm{Ga}_{2} \mathrm{O}_{3}$ 开始分解为 $\mathrm{Ga}_{2} \mathrm{O}$ 和 $\mathrm{Ga}, \mathrm{NH}_{3}$ 分子也逐步分解为 $\mathrm{NH}_{2} 、 \mathrm{NH}$ 和 $\mathrm{N}, \mathrm{Ga}_{2} \mathrm{O}$ 与 $\mathrm{NH}_{3}$ 反应生成 $\mathrm{GaN}, \mathrm{N}$ 原子 和 $\mathrm{Ga}$ 原子直接反应也生成 $\mathrm{GaN}$. 这些 $\mathrm{GaN}$ 分子扩 散、凝聚, 并与其他的 $\mathrm{GaN}$ 分子聚集形成 $\mathrm{GaN}$ 微晶. 随着氨化时间的延长, 微小的 $\mathrm{GaN}$ 晶体逐步长大, 从而成为一维 $\mathrm{GaN}$ 纳米结构. 同时, 由于实现 $\mathrm{Mg}$ 掺杂的难点在于 $\mathrm{GaN}$ 材料的生长温度是 $800{ }^{\circ} \mathrm{C}$ 以 上, 而 $\mathrm{Mg}$ 在 $600{ }^{\circ} \mathrm{C}$ 时就已经开始挥发了. 因此它们 很难得到一个合适的交叉温度. 我们则选择类似 Delta 掺杂方法, $\mathrm{Ga}$ 原子和 $\mathrm{Mg}$ 原子层层相隔, 使得 在生长过程中 $\mathrm{Mg}$ 原子有更多的机会注人 $\mathrm{GaN}$ 单 晶中. 虽然无法避免 $\mathrm{Mg}$ 的挥发, 但还是有效地实现

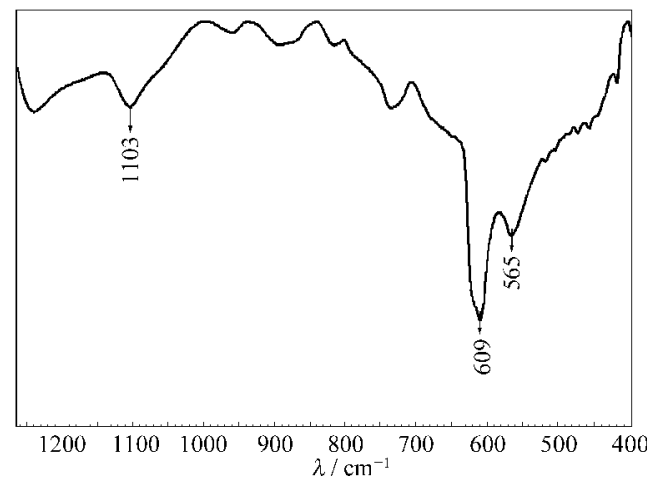

图 $5 \mathrm{Mg}$ 掺杂 $\mathrm{GaN}$ 纳米线的傅里叶红外吸收光谱图

Fig.5 The FTIR spectra of Mg-doped GaN nanowires
了 $\mathrm{GaN}$ 纳米线的 $\mathrm{Mg}$ 掺杂. 相关化学反应式如下:

$$
\begin{aligned}
& 2 \mathrm{NH}_{3}(\mathrm{~g}) \rightarrow \mathrm{N}_{2}+3 \mathrm{H}_{2}(\mathrm{~g}) \\
& \mathrm{Ga}_{2} \mathrm{O}_{3}(\mathrm{~s})+2 \mathrm{H}_{2}(\mathrm{~g}) \rightarrow \mathrm{Ga}_{2} \mathrm{O}(\mathrm{g})+2 \mathrm{H}_{2} \mathrm{O}(\mathrm{g}) \\
& \mathrm{Ga}_{2} \mathrm{O}(\mathrm{g})+2 \mathrm{NH}_{3}(\mathrm{~g}) \rightarrow 2 \mathrm{GaN}(\mathrm{s})+2 \mathrm{H}_{2}(\mathrm{~g})+\mathrm{H}_{2} \mathrm{O}(\mathrm{g})
\end{aligned}
$$

\section{3 结 论}

采用类似 Delta 掺杂方法在 $850{ }^{\circ} \mathrm{C}$ 时成功合成 了 $\mathrm{Mg}$ 掺杂 $\mathrm{GaN}$ 单晶纳米线. SEM 和 TEM 的结果 表明, 制备的 $\mathrm{GaN}$ 纳米线表面粗粘, 直径约为 35 $\mathrm{nm}$, 长度可达几十微米. XRD、FTIR 和 SAED 的结 果说明, 纳米线为六方纤锌矿结构的 $\mathrm{GaN}$ 单晶体. $\mathrm{EDX}$ 分析表明, GaN 纳米线中含有约 $3 \%$ (原子分 数)的 Mg.

\section{References}

1 Fasol, G. Science, 1996, 272: 1751

2 Nakamura, S. Science, 1998, 281: 956

3 Brown, S. A.; Reeves, R. J.; Haase, C. S. Appl. Phys. Lett., 1999, 75: 3285

4 Nam, T. H.; Kim, J. H.; Kim, T. Y. J. Mater. Sci. Lett., 2002, 21: 1851

5 Zhou, S. M. Phys. E, 2006, 33: 394

6 Wang, Q.; Sun, Q.; Jena, P. Phys. Rev. Lett., 2005, 95: 167202

7 Han, S. E.; Oh, H.; Kim, J. J.; Seong, H. K.; Choi, H. J. Appl. Phys. Lett., 2005, 87: 062102

8 Zhou, S. M. Physical Letters A, 2006, 357: 374

9 Dong, Z. H.; Xue, C. S.; Zhuang, H. Z. Phys. E, 2005, 27: 32

10 Han, D. S.; Park, J.; Rhie, K. W.; Kim, S.; Chang, J. Appl. Phys. Lett., 2005, 86: 032506

11 Cai, X. M.; Djurišiæ, A. B.; Xie, M. H.; Chiu, C. S.; Gwo, S. Appl. Phys. Lett., 2005, 87: 183103

12 Boo, J. H.; Rohr, C.; Ho, W. J. Crys. Grow., 1998, 189/190: 439

13 Sun, Y.; Miyasato, T.; Wigmore, J. K. J. Appl. Phys., 1999, 85: 3377

14 Melendez, L. M.; Menendez, J.; Kramer, K. M. J. Appl. Phys., 1997, 82: 4246

15 Meng, G. W.; Zhang, L. D.; Qin, Y. Nanostruct. Mater., 1999, 12 : 1003 